\title{
The problem of creating an automated system to control growth of single crystal sapphires from melt as a problem of control and monitoring of a complex nonlinear and dynamic system
}

\author{
$V A$ Petrosyan $^{1, *}, A V$ Belousov $^{2}, A G$ Grebenik $^{2}$ and $Y u A$ Koshlich $^{2}$ \\ ${ }^{1}$ LLC "Techsapphire", Belgorod, Russia \\ ${ }^{2}$ BSTU named after V.G. Shukhov, Belgorod, Russia
}

\begin{abstract}
The paper mentions some problems of automated control system development for growth of large (150 kg and above) single crystal sapphires. We obtain an analytical equation for the temperature distribution and thermal stresses along the crystal axis during the growth phase. An analysis was carried out and numerical estimates were obtained for the axial distribution of components of thermoelastic stresses depending on the physical, optical, and geometric parameters of the crystal. It is shown that the cause of thermal stresses and blocks during crystal growth is the nonlinear temperature dependence of thermal conductivity and thermal expansion coefficients.
\end{abstract}

\section{Introduction}

Based on the general concepts of the theory of normal directional crystallization from the melt, it is known that the crystallization rate $v_{c r}$ is proportional to the supercooling of the melt $\Delta T$ or the temperature gradient at the phase interface. That is, $v_{c r} \sim \Delta T$ or $v_{c r} \sim \operatorname{grad} T$.

In the harmonic approximation for the growth rate, the expression:

$$
v_{c r}=v_{0}+v_{1} \sin (\omega t+\varphi)
$$

where $v_{0}$ is an average crystal growth rate, $v_{1}$ is an amplitude of change in growth rate due to temperature fluctuations in the melt near the phase transition boundary, $\omega$ is a temperature oscillation frequency, $t$ is time, $\varphi$ is some phase. Based on equation (1), we can determine the magnitude of the dynamic supercooling or overheating of the melt at the crystallization front:

$$
\Delta T=\Delta T_{0}+B \sin (\omega t+\varphi)
$$

where $\Delta T_{0}$ is a fixed value of "physical supercooling" (the difference between the melting point and the crystallization temperature), $B$ is a supercooling change amplitude.

It can be seen from equation (2) that, depending on the amplitude and phase of the change in the supercooling, periodic localized melting of the crystal at the crystallization front can occur with re-crystallization of the melted layer.

It should be noted that temperature fluctuation in individual local areas on the crystallization front may occur due to mixing of hotter and less hot melt layers at a forced convention and with different density of melt saturation with gas inclusions in these zones. As a result, in the places of melting, more favorable conditions arise for the capture of micron-sized bubbles and their further growth into a single crystal.

It follows from the above that an important conclusion is that the magnitude of the supercooling at the crystallization front determines not only the conditions for the growth of a single crystal, but also its perfection.

We need to note one more important fact for the controlling systems of the growth rate and maintaining stability at the crystallization front. The fact is that for sapphire the melting point and the crystallization temperature do not coincide. The melting point is well reproduced at different heating rates, and the crystallization temperature depends on the cooling rate of the melt [1]. Therefore, in formula (2), $\Delta T_{0}$ should be understood as the average value of "physical supercooling".

It follows from the above that fluctuations in the magnitude of dynamic supercooling affect the change in the rate of crystallization and lead to stricter requirements for the system for stabilizing gradients at the crystallization front of $\mathrm{Al}_{2} \mathrm{O}_{3}$, than, for example, for silicon.

We will now define two cases of dynamic supercooling. The first is small fluctuations in supercooling, within the zone of "physical supercooling", which are stochastic in nature and depend on many factors. These processes are described by methods of nonlinear dynamics, in particular, the theory of dynamic or deterministic chaos.

The second case is high-amplitude supercooling oscillations that go beyond the limits of "physical

Corresponding author: iitusnik@gmail.com 
supercooling". These fluctuations can occur during growth control using an automated system that operates on a feedback signal from a weight sensor and regulates the power at the heater.

Since dynamic weighing is an indirect method of controlling crystal growth, it becomes necessary to use the so-called observation equation, which relates the force acting on the weight sensor with the required crystal mass and the rate of its crystallization.

\section{The problem of controlling the crystal growth using the feedback signal from the weight sensor}

When crystals are grown by the modified Kyropoulos method (GOI method), a significant part of the crystal is in the melt and a pushing force acts on the crystal. The magnitude of this force depends on the fluctuation of the melt density depending on the temperature, variations in the crystallization rate, changes in the angle of growth. It is also necessary to add the dependence of the weight sensor readings on fluctuations in the temperature of its environment, on various kinds of vibrations, etc. Of all these parameters, we distinguish two main ones-the linear crystallization rate (the vertical component of the rate of front advancement into the melt during growth) and the shape of the crystallization front, depending on the angle of the growth cone. The weight sensor signal is a function of these two parameters and the cause of the change in weight gain can be either a change in the linear crystallization rate or a change in the angle at the apex of the growth cone. In view of this, the control object is not observable in a fairly wide "dead zone" [24].

With this in mind, as well as the inertia of heat transfer from the heater to the melt, there is a loss of controllability and the control system can enter the selfoscillatory mode. During the growth process, turbulent flows in the melt, vortex flows at the crystallization front, etc. can occur.

There are two main ways to stabilize a chaotic system: without feedback and with feedback. The first is called chaos suppression, and the second is chaos control. The second method which includes active control will be described in future research.

The task of searching for controlling external influences, which make it possible to minimize temperature fluctuations at the crystallization front, can be formulated in a general form. Suppose that in the system under consideration there are two competing processes: a nonlinear source $Q(T)$, which reflects feedback, and a dissipative source, nonlinearity of which is determined by the coefficient $K(T)$. If these functions have a power form, then the system can be described by the equation:

$$
\frac{\partial T}{\partial t}=\frac{\partial}{\partial x}\left(K(T) \frac{\partial T}{\partial x}\right)+Q(T)
$$

This model is known from studies of plasma physics and the theory of controlled thermonuclear fusion and is called the model of complex thermal structures. The complex structures in this model are unstable and can exist only with the correct specification of the initial data. In [5], we have investigated a similar model when calculating the temperature distribution along the crystal axis during growth and optimized some process parameters based on identification with experimental data.

In this case, we were guided by the fact that the parameters $Q(T)$ and $K(T)$ in the equation (3) have a different physical interpretation depending on the optical thickness of the layer $\tau=\beta_{0} L$ (where $\beta_{0}$ is the attenuation coefficient, $L$ is the thickness of the layer), which spreads the flow of heat.

\section{The role of sapphire crystal transparency and the effect of nonlinear temperature parameters on the quality of crystals}

In the general case, the nonstationary problem of joint radiation-conductive heat transfer in the direction of the $X$ axis in a layer of a semi-transparent medium in terms of dimensionless parameters can be written as [6]:

$$
\frac{\partial \theta(\tau, \xi)}{\partial \xi}=\frac{\partial^{2} \theta(\tau, \xi)}{\partial \tau^{2}}-\frac{1}{N^{*}} \frac{\partial^{2} Q_{r}(\theta, \xi)}{\partial \tau^{2}}+H(\tau, \xi)
$$

where $\theta(\tau, \xi)$ is a temperature, $Q_{r}(\theta, \xi)$ is a radiation flux density, $H(\tau, \xi)$ is a bulk power of internal sources, $N^{*}$ is a conductive-radiation parameter.

In this equation, the parameter $N^{*}$ characterizes the relative contribution of thermal conductivity compared to radiation. This parameter has large values when thermal conductivity prevails, and small ones when radiation predominates. That is, when the medium is only scattering $\left(N^{*} \rightarrow \infty\right)$ and the radiation term in equation (4) disappears, then it turns into the usual heat conduction equation.

For a medium with a high optical thickness, the attenuation coefficient $\beta_{0}$ is replaced by the absorption coefficient. That is, for a sapphire crystal, $K(T)$ is the radiative thermal conductivity coefficient in the diffusion approximation (Rosseland approximation [6]). The expression for this coefficient is [6-8]:

$$
K_{S L}=\frac{16 N^{2} \sigma T_{S L}^{3}}{3 \beta}
$$

where $T_{S L}$ is a crystallization temperature, $\sigma$ is a StefanBoltzmann constant, $N$ is a refraction coefficient.

If we calculate the conducted-radiation parameter $N^{*}=\frac{K_{F} \beta_{0}}{4 N^{2} \tau T_{S L}^{3}}$ for sapphire, then with $K_{F}=3 \frac{\mathrm{W}}{\mathrm{m} \cdot \mathrm{K}}, \beta_{0}=$ $0.35 \frac{1}{\mathrm{~cm}}, \quad N=1.79, \quad T_{S L}=2308 \mathrm{~K}^{\circ} \quad$ and $\quad \sigma=5.67$. $10^{-8} \frac{W}{m^{2} K^{4}}$ will get $N^{*}=0.0118$. 
Thus, in solving the problem of temperature distribution along the axis of a growing crystal, the nonlinear parameter $K_{S L}(T)$ plays a significant role.

In [5], we obtained expressions for the temperature distribution along the axis of a growing crystal depending on the geometric and nonlinear parameters $Q_{1}(T)$ [9] and $K_{S L}^{R}(T)$. Based on this, the formulas for calculating the temperature gradient at the crystallization front and the maximum crystallization rate were obtained:

$$
\begin{gathered}
\left|\operatorname{grad} \overline{T_{S}}(x)\right|_{x \rightarrow 0}=\frac{T_{S L}}{2 N} \sqrt{\frac{3 \varepsilon_{\text {eff }} \beta_{S L} \sqrt{\sin \left(\frac{\alpha}{2}\right)}}{D_{S}}} \\
v_{\max }=\frac{8 N \sigma T_{S L}^{4}}{3 \rho_{S} Z} \cdot \sqrt{\frac{3 \varepsilon_{\text {eff }} \sqrt{\sin \left(\frac{\alpha}{2}\right)}}{\beta_{S L} D_{S}}}
\end{gathered}
$$

Taking into account the numerical values for the Stefan-Boltzmann constant $\sigma=5.67 \cdot 10^{-8} \frac{W}{m^{2} K^{4}}$, the specific latent heat of fusion $Z=683.2 \frac{\mathrm{W} \cdot \mathrm{h}}{\mathrm{kg}}$, the melt absorption coefficient $\beta_{S L}=0.3 \div 0.9 \frac{1}{\mathrm{~cm}}$, with a fixed crystal diameter $D_{S}=0.3 \mathrm{~m}$ and a corner of the growth cone $\alpha=180^{\circ}$ numerical values can be obtained for temperature gradients at the crystallization front and maximum growth rate. The results of the calculations are given in tables 1 and 2:

Taking into account the results obtained in [5], it is possible to conduct a qualitative analysis and compare the role of various factors in the formation of the thermally stressed state of a growing crystal.

Taking into account the formula for temperature distribution along the $X$ axis [5], it is possible to calculate the axial component of thermal stresses using the formula [8]:

$$
\Sigma_{x}=\frac{\alpha_{T} E x^{2}}{(1-\mu)} \cdot \frac{d^{2} T_{S L}(x)}{d x^{2}}
$$

where $\alpha_{T}$ is a thermal expansion coefficient, $\mu$ is a Poisson's ratio, $E$ is Young's modulus.

Substituting $T_{S L}(x)$ from [5] into (8) can be obtained:

$$
\begin{aligned}
& \Sigma_{x}=\frac{T_{S L}(0) \cdot E \alpha_{T} \varepsilon_{e f f} \beta_{S L} x^{2} \sqrt{\sin \left(\frac{\alpha}{2}\right)}}{2 N^{2}(1-\mu) D_{S}} . \\
& \cdot \exp \left(\frac{-x}{2 N} \sqrt{\frac{2 \varepsilon_{e f f} \beta_{S L} \sqrt{\sin \left(\frac{\alpha}{2}\right)}}{D_{S}}}\right)
\end{aligned}
$$

The calculation results are shown as graph $1\left(\frac{\alpha}{2}=\right.$ $\left.90^{\circ}\right)$ and graph $2\left(\frac{\alpha}{2}=45^{\circ}\right)$ in Figure 1, taking into account the numerical values of the parameters $N=$ $1.79, \quad T_{S L}=2308 \mathrm{~K}^{\circ}, \quad \mu=0.27, \quad E=4.34 \cdot 10^{5} \mathrm{MPa}$ [10], $\beta_{S L}=24.4 \frac{1}{m}, \frac{\alpha}{2}=90^{\circ}, \frac{\alpha}{2}=45^{\circ}, \quad \alpha_{T}=5.58$. $10^{-6} \frac{1}{K^{\circ}}[1]$.

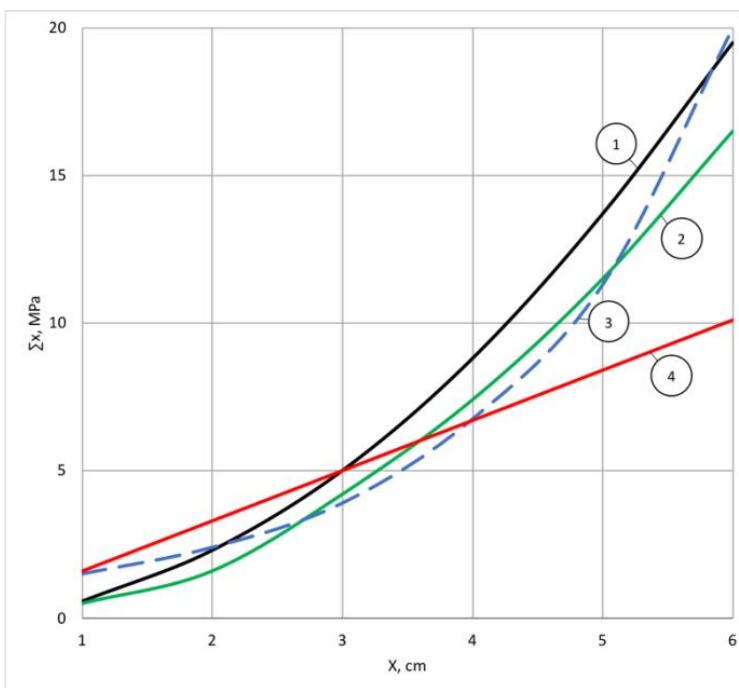

Fig. 1. Distribution of components of thermal stress tensor.

Table 1. Numerical values for temperature gradients at the crystallization front.

\begin{tabular}{|c|c|c|c|}
\hline \multirow{4}{*}{$\operatorname{grad}_{S}(T) \mid$} & $\begin{array}{c}\text { Melt absorption coefficient } \beta_{S L} \\
{\left[\mathrm{~cm}^{-1}\right]}\end{array}$ & Angle of growth cone $\alpha$ \\
\cline { 2 - 3 } & 4.02 & 0.3 & \multirow{2}{*}{$180^{\circ}$} \\
\cline { 2 - 3 } & 5.2 & 0.5 & \\
\cline { 2 - 3 } & 5.6 & 0.6 & \\
\cline { 2 - 3 } & 10 & 0.9 & ${ }^{\circ} \mathrm{C}$ \\
\cline { 2 - 3 }
\end{tabular}

Table 2. Numerical values of the maximum growth rate.

\begin{tabular}{|c|c|c|c|}
\hline \multirow{4}{*}{$v_{\max }$} & Growth rate $\left[\frac{\mathrm{mm}}{\mathrm{h}}\right]$ & $\begin{array}{c}\text { Melt absorption coefficient } \beta_{S L} \\
{\left[\mathrm{~cm}^{-1}\right]}\end{array}$ & Angle of growth cone $\alpha$ \\
\cline { 2 - 3 } & 6.08 & 0.3 & \multirow{2}{*}{$180^{\circ}$} \\
\cline { 2 - 3 } & 4.65 & 0.5 & \\
\cline { 2 - 3 } & 4.25 & 0.6 & 0.9 \\
\hline
\end{tabular}


In the same figure, the dashed line (graph 3) shows the critical shear stress for the sapphire easy slip system [10] (extrapolation of data from [11]).

In [10], it was shown that in the case of a nonlinear quadratic temperature dependence of the thermal expansion coefficient $\alpha_{T}(T)$, the normal components of the thermoelastic stress tensor turn out to be linear functions of the axial coordinate.

Graph 4 in Figure 1 shows the linear variation of stresses for the case of sapphire growth with length $L=$ $100 \mathrm{~mm}$ and diameter $D_{S}=10 \mathrm{~mm}$ in a linear temperature field [10].

To preserve the scale and display in one picture, we used the same parameters when calculating using expression (9).

From Figure 1 it can be seen that the values of thermal stresses (graphs 1,2 and 3) exceed or are very close to critical values in the range up to $\mathrm{x}=58 \mathrm{~mm}$. This means that defects in the form of blocks, twins and cracks are possible in this range. Thus, the nonlinear nature of the dependence of the thermal expansion coefficient causes blocks to appear in the upper part of the crystal as it grows to a predetermined diameter after seeding, that is, it ultimately affects the quality of the crystal. This is especially important when growing large crystals using the GOI method. The appearance of blocks in the upper part of the crystal was observed in experiments performed at Techsapphire LLC (Belgorod), when growing crystals weighing $100 \mathrm{~kg}$ at different temperatures of growing the cone of growth.

\section{Conclusion}

Based on the analysis of work on the development of GOI and Czochralski [12-14] methods for growing single crystals from the melt, we can conclude that, to date, there is no fully automated, strictly replicable technology for the production of defect-free single crystals. For single crystal sapphires, this is primarily optical symmetry, the absence of residual thermal stresses and blocks, the absence of bubbles and impurities. This is not related to the technical capabilities of creating automated growth process control systems.

One of the reasons for the appearance of defects is that when the growth is controlled by a feedback signal from a weight sensor, a zone of uncontrollability appears and a random change in the crystallization temperature regime occurs. In addition, at high temperatures, the melts of oxides and semiconductors have a different character of chemical interaction with molybdenum or tungsten screens, crucible material, etc., and since the growth of single crystals can occur both in vacuum and in a reducing or neutral gas atmosphere, there is a need to work out new technological solutions and temperature regimes in the process of growth.

However, the main reason is of a fundamental nature and, as noted in this article, is associated with a nonlinear temperature dependence of some physical and thermodynamic parameters of sapphire. In particular, residual thermal stresses arise in the crystal, which under certain growth conditions can lead to the appearance of blocks in the crystal and even to its cracking.

In turn, the Poynting vector $[15,16]$ is responsible for the optical symmetry of the crystal (a cruciform conoscopic picture). Poynting vector is associated with electromagnetic parameters and components of the dielectric constant tensor, which depend on the components of the thermal stress tensor. Based on this, it was concluded [15] that the growth of crystals from the melt using the GOI method, when the crystal is hanging on a moving rod, has a fundamental limitation on the size and mass of the crystal. That is, the larger the mass of the grown crystal, the more the conoscopic picture is distorted and its optical symmetry is broken.

\section{References}

1. K.L. Lobatsevich, Improving the stability of the rate of crystallization of monocrystals of leucosapphire by the Kyropoulos method by introducing predictive control on the rate of change of mass of the single crystal, Thesis for the degree of candidate of technical sciences (2010)

2. A.V. Borodin, Equipment for growing sapphire single crystals by the Kyropoulos method with a device for dynamic crystal weighting and automatic control with feedback, Scientific instrument engineering, 24(3), 92-98 (2014)

3. A.V. Belousov, Y.A. Koshlich, A.G. Grebenik, About one approach to the automation of the process of seeding the synthetic sapphire single crystals by the Kyropoulos method, The Bulletin of BSTU named after V.G. Shukhov, 3, 128-133 (2017)

4. A.G. Grebenik, M.V. Panchenko, S.A. Karatach, Improving the efficiency of the process of seeding single crystals of artificial sapphire on the basis of technical vision using the method of selection of active contours, News of South-West State University. Management series, computer technology, computer science. Medical Instrument, 8-3(28), 41-49 (2018)

5. V.A. Petrosyan, A.V. Belousov, A.G. Grebenik, Solution of the Stefan problem during radiationconductive heat transfer in the process of growing sapphire single crystals by a modified Kyropoulos method, International Seminar on Electron Devices Design and Production (SED) (2019)

6. M.N. Oscisk, Complicated heat transfer, 606 (1976)

7. Kh.S. Bagdasarov, High temperature crystallization from melt, 160 (2004)

8. S.P. Malyukov, Yu.A. Klunnikova, The calculated model of the distribution of temperature fields in sapphire single crystals, Proceedings of the IV International Scientific Conference "JCSS-2011", 259-264 (2011)

9. I.I. Ignatov, Temperature growth conditions of corundum as related to crystal perfection, Theoretical calculation Journal of Crystal Growth, 52, 411-416 (1981) 
10. S.I. Bakholdin, E.V. Galaktionov, E.A. Tropp, Calculation of thermal stresses in profiled single crystals, taking into account the temperature dependence of thermal expansion, Technical Physics Journal, 60, 11 (2010)

11. K. Peter, D. Lagerlof, A.H. Heuer, Slip and Twinning in Sapphire $(\alpha-\mathrm{Al} 2 \mathrm{O} 3)$ J.Am, Ceram, 77(2), 385 (1994)

12. V.A. Tatarchenko, V.A. Antonovich, Sustained crystal growth, 240 (1988)

13. E.R. Dobrovinskaya, L.A. Litvinov, V.V. Pishik, Encyclopedia of Sapphire, 508 (2004)

14. W. Bardsley, G. Green, C. Holliday, D. Hurle, Automatic control of Czochralski crystal growth J, Crystal Growth, 16, 277-279 (1972)

15. A.A. Abgaryan, Modeling of temperature and thermoelastic fields in sapphire in threedimensional curvilinear coordinates, Mat Modeling, 13(8), 20-30 (2001)

16. L.D. Landau, E.M. Lifshits, Electrodynamics of Continuous Media, A Course of Theoretical Physics, 8, 620 (1982) 